\title{
A SEARCH FOR ALIQUOT CYCLES AND AMICABLE PAIRS
}

\author{
DAVID MOEWS AND PAUL C. MOEWS
}

\begin{abstract}
A search for aliquot cycles below $3.6 \cdot 10^{10}$ and amicable pairs below $10^{11}$ is described. Three new cycles of length 4 and one new cycle of length 6 are exhibited. Four triples of amicable pairs with the same pair-sum are also exhibited.
\end{abstract}

The idea of an aliquot cycle is an extension of the concepts of perfect numbers and amicable pairs, which are aliquot cycles of lengths 1 and 2, respectively. Let $\sigma(n)$ be the sum of the divisors of $n$, where $n$ is a natural number. Define $s(n)$ to be the sum of the divisors of $n$ exclusive of $n$, that is, $s(n)=\sigma(n)-n$. An aliquot cycle of length $k$ is then a finite sequence of distinct natural numbers $\left(a_{1}, \ldots, a_{k}\right)$ such that $a_{1}=s\left(a_{k}\right)$, and for each $i=1, \ldots, k-1, a_{i+1}=$ $s\left(a_{i}\right)$. In an earlier paper [7] we described a search for aliquot cycles that covered some of the cycles with length 32 or less and smallest element below $10^{10}$. A. Flammenkamp [5] recently reported a search for aliquot cycles that covered some of the cycles with length 50 or less and smallest element below $5 \cdot 10^{9}$. To date, including the results of these searches, 35 cycles with length at least 3 are known, 30 of length 4 , two of length 8 , and one each of lengths 5,9 , and $28[1,2,4,5,7,8,13]$. In this paper we describe a new search to find all aliquot cycles, of any length, such that the element preceding the largest element of the aliquot cycle does not exceed $3.6 \cdot 10^{10}$, and all amicable pairs with smaller element not exceeding $10^{11}$.

In the aliquot cycle search, the method used was similar to that used in the third search in [7]. For all $a_{1}$ between 2 and $3.6 \cdot 10^{10}, a_{2}=s\left(a_{1}\right)$ was constructed. The number $a_{2}$ was then the putative largest member of the aliquot cycle; hence if $a_{2}$ was less than $a_{1}$, the search stopped immediately. Otherwise, $a_{i}=s\left(a_{i-1}\right)$ was constructed for $i=3,4$, and so on. If $a_{i}$ ever became equal to $a_{1}$, iteration stopped and a new aliquot cycle was recorded. On the other hand, if $a_{i}$ became equal to $1, a_{i}$ exceeded $a_{2}$, the sequence of $a_{i}$ 's fell into a cycle of length dividing 4 not involving $a_{1}, a_{i}$ equalled 12496, the smallest member of the only known aliquot cycle of length 5 , or $a_{i}$ equalled 14316 , the smallest member of the only known aliquot cycle of length 28 , iteration stopped and a new cycle was not recorded. If $i$ became equal to 5001, iteration stopped

Received by the editor May 19, 1992.

1991 Mathematics Subject Classification. Primary 11A25; Secondary 11-04.

Key words and phrases. Amicable numbers, sociable numbers, aliquot cycles.

We wish to thank the University of Connecticut Computer Center for providing grants for lowpriority computation on their IBM ES 9000-580. This material is based on work supported under a National Science Foundation Graduate Fellowship. 
TABLE 1. New aliquot cycles with length at least 3

$1 \quad\left(15837081520=2^{4} \cdot 5 \cdot 13 \cdot 263 \cdot 57901\right.$, $26042149708=2^{2} \cdot 37 \cdot 137 \cdot 1284383$,

$2 \quad\left(17616303220=2^{2} \cdot 5 \cdot 79 \cdot 227 \cdot 49117\right.$, $25854330388=2^{2} \cdot 17 \cdot 1123 \cdot 338567$,

$3 \quad\left(21548919483=3^{5} \cdot 7^{2} \cdot 13 \cdot 17 \cdot 19 \cdot 431\right.$, $24825443643=3^{2} \cdot 7^{2} \cdot 11 \cdot 13 \cdot 19 \cdot 20719$, $25958284443=3^{2} \cdot 7^{2} \cdot 13 \cdot 19 \cdot 167 \cdot 1427$,

$4 \quad\left(21669628904=2^{3} \cdot 7 \cdot 11 \cdot 35177969\right.$, $25367088104=2^{3} \cdot 29 \cdot 179 \cdot 610843$,

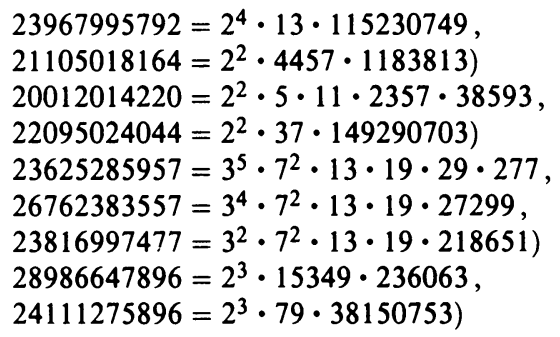

and the sequence was examined by hand. In all these latter cases, 153 in all, it was found that the sequence had fallen into a known aliquot cycle of length 8 or 9.

As discussed in [7], the computation of $s\left(a_{1}\right)$ can be sped up using a sieving method, and the computation of subsequent $s\left(a_{i}\right)$ 's, which must fall into a specified interval if the computation is to continue, can be sped up by noting that a partial factorization of $a_{i}$ is sometimes enough to show that $s\left(a_{i}\right)$ falls outside an interval. Searching for aliquot cycles of arbitrary length is successful because the average length of the aliquot sequence which must be searched for each integer is small. Each new member of the aliquot sequence requires the factorization of the previous member. For integers near $10^{9}$ the average number of factorizations required per integer was 2.40 , while for integers near $2.5 \cdot 10^{10}$, the average number of factorizations required was 2.53 .

The search was performed on an IBM ES 9000-580 and took about 2500 CPU hours. Besides already-known aliquot cycles, three cycles of length 4 and a cycle of length 6 were the only aliquot cycles found with length at least 3 . These cycles are shown in Table 1 . We believe this to be the first example of a cycle of length 6 .

The search just described found all amicable pairs with smaller member less than $3.6 \cdot 10^{10}$. The search was continued to find all amicable pairs with smaller member less than $10^{11}$, taking approximately 350 hours on the ES 9000-580. For all $a_{1}$ between $3.6 \cdot 10^{10}$ and $10^{11}$ not divisible by six, $a_{2}=s\left(a_{1}\right)$ was computed. If $a_{2}$ was less than $a_{1}, s\left(a_{2}\right)$ was not computed, since $a_{1}$ was supposed to be the smaller member of the amicable pair. If $a_{1}$ and $a_{2}$ were both even and $a_{2}$ was twice $a_{1}$ or more, $\left(a_{1}, a_{2}\right)$ could not have been an amicable pair [6], so $s\left(a_{2}\right)$ was not computed. Otherwise, $s\left(a_{2}\right)$ was checked for equality to $a_{1}$, possibly giving a new amicable pair. If $a_{1}$ was divisible by six, an amicable pair $\left(a_{1}, a_{2}\right)$ would have had to have been of the form $\left(2^{\mu} M^{2}, N^{2}\right)$ for some $\mu$ and odd $M$ and $N$ [9]. For all $a_{1}$ less than $10^{11}$ divisible by six and equal to a square or twice a square, $s\left(a_{1}\right)$ was checked to see if it was a perfect square, which it never was. Hence no amicable pairs with smaller member divisible by six were found.

In all, a total of 3340 amicable pairs with smaller member less than $10^{11}$ were found. A list of all amicable pairs with smaller member less than $10^{10}$ [9] contains 1427 of these. Of the remaining 1913 pairs, 419, those with smaller member between $10^{10}$ and $2 \cdot 10^{10}$, were computed by te Riele in a 1990 search [10], 275 can be found in [12], three in [9], two in [3], and 40 in an unpublished list [11]. (Some can be found in more than one of these places.) We expect that many of the remaining 1263 are new. C. Pomerance [9] has asked whether there 
TABLE 2. Triples of amicable pairs with the same pair-sum

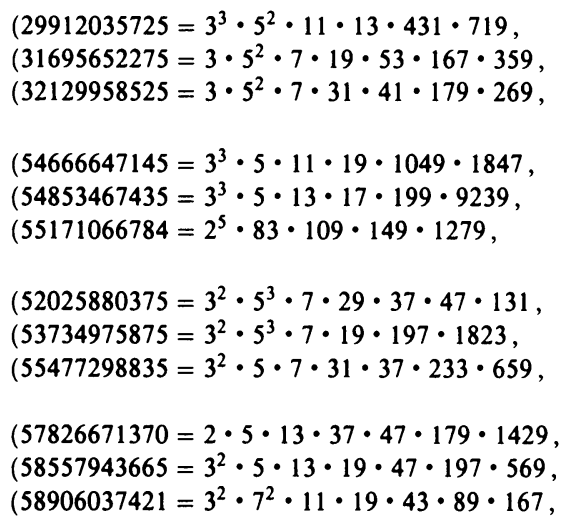

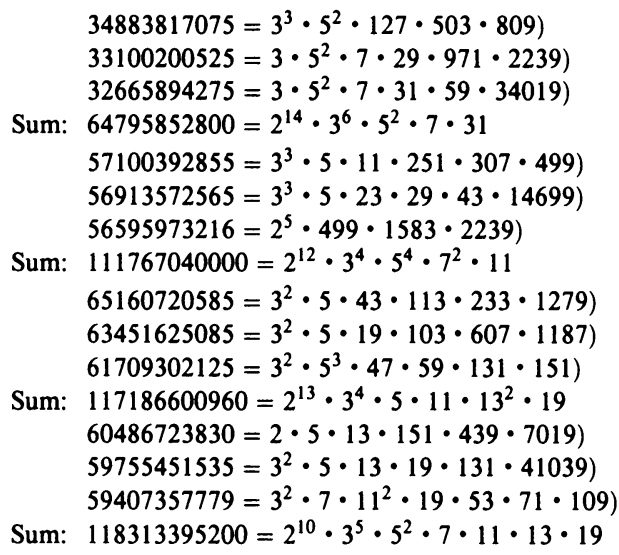

exist pairs, triples, and so forth of amicable pairs having the same pair-sum. In [9], 37 pairs of amicable pairs having the same pair-sum are listed. We believe that we have located the first triple of amicable pairs with the same pair-sum. In fact, we found four triples in all, shown in Table 2.

There were, in all, 70 pairs of amicable pairs with the same pair-sum in our list of pairs. The pair with the smallest ratio of smaller member to larger member was

$$
\begin{aligned}
(35049418250 & =2 \cdot 5^{3} \cdot 7^{2} \cdot 11 \cdot 23 \cdot 43 \cdot 263, \\
54192685558 & =2 \cdot 11 \cdot 191 \cdot 967 \cdot 13337),
\end{aligned}
$$

with ratio 0.646756 , and the pair with the largest ratio was

$$
\begin{aligned}
(85617896265 & =3^{4} \cdot 5 \cdot 11 \cdot 53 \cdot 349 \cdot 1039, \\
85625175735 & \left.=3^{4} \cdot 5 \cdot 11 \cdot 59 \cdot 199 \cdot 1637\right),
\end{aligned}
$$

with ratio 0.999915 .

The authors will provide interested parties with a list of amicable pairs found upon request. In addition, we have deposited this list in the UMT file.

Added in proof. We have since extended our aliquot cycle search to cover all cycles with member preceding the largest not exceeding $6.5 \cdot 10^{10}$. Nothing new was found except for a 4-cycle with smallest member 44379752648 .

\section{BIBLIOGRAPHY}

1. W. Borho, Über die Fixpunkte der $k$-fach iterierten Teilersummenfunktion, Mitt. Math. Gesellsch. Hamburg 9 (1969), 34-48.

2. H. Cohen, On amicable and sociable numbers, Math. Comp. 24 (1970), 423-429.

3. P. Costello, Amicable pairs of the form $(i, 1)$, Math. Comp. 56 (1991), 859-865.

4. J. S. Devitt, R. K. Guy, and J. L. Selfridge, Third report on aliquot sequences, Proc. 6th Manitoba Conf. Numerical Math. 1976, Congr. Numer. XVIII, Utilitas Math., Winnipeg, Manitoba, 1977 , pp. 177-204. 
5. A. Flammenkamp, New sociable numbers, Math. Comp. 56 (1991), 871-873.

6. E. J. Lee and J. S. Madachy, The history and discovery of amicable numbers-Part I, J. Recreational Math. 5 (1972), 77-93.

7. D. Moews and P. C. Moews, A search for aliquot cycles below $10^{10}$, Math. Comp. 57 (1991), 849-855.

8. P. Poulet, \#4865, L'intermédiare des math. 25 (1918), 100-101.

9. H. J. J. te Riele, Computation of all the amicable pairs below $10^{10}$, Math. Comp. 47 (1986), 361-368.

10. _ personal communication, Dec. 9, 1991.

11. _ personal communication, Jan. 28, 1992.

12. H. J. J. te Riele, W. Borho, S. Battiato, H. Hoffmann, and E. J. Lee, Table of amicable pairs between $10^{10}$ and $10^{52}$, Centrum voor Wiskunde en Informatica, Note NM-N8603, Stichting Math. Centrum, Amsterdam, 1986.

13. S. C. Root, Item 61, M.I.T. Artificial Intelligence Memo no. 239, Feb. 29, 1972.

Department of Mathematics, University of California, Berkeley, California 94720

E-mail address: moews@math.berkeley.edu

Institute of Materials Science, University of Connecticut, Storrs, Connecticut 06269

E-mail address: moews@uconnvm.bitnet 